\title{
A note on symmetry breaking in a non linear marketing model
}

\author{
Andrea Caravaggio $^{1}$ • Lorenzo Cerboni Baiardi ${ }^{2}$ - Mauro Sodini ${ }^{3,4}$
}

Received: 30 January 2021 / Accepted: 1 June 2021 / Published online: 27 July 2021

(c) The Author(s) 2021

\begin{abstract}
In this paper, we consider the nonlinear discrete-time dynamic model proposed by Bischi and Baiardi (Chaos Solitons Fractals 79:145-156, 2015a). The model considers players with adaptive adjustment mechanisms towards the best reply and a form of inertia in adopting such mechanism. Moreover, we formulate an extension of the original model, where endogenous market size is considered. Through numerical simulations, we show that multiple attractors may exist in the presence of homogeneous agents and the emergence of non-synchronized trajectories both in the short (on-off intermittency) and long (global riddling) run. Therefore, the article highlights that strategic contexts exist in which the players' knowledge of the market and the adoption of the best reply do not always allow the use of the representative agent's rhetoric to describe the dynamics of the model.
\end{abstract}

Keywords Nonlinearity $\cdot$ Synchronization $\cdot$ Market share models $\cdot$ Representative agent

JEL Classification C61 · C62

\section{Introduction}

In recent years the economic theory has become increasingly interested in the study of contexts characterized by the presence of heterogeneous agents both in the macroeconomic, microeconomic, and financial spheres. Several modeling approaches have

$\bowtie \quad$ Lorenzo Cerboni Baiardi

lorenzo.cerboni@unical.it

1 Department of Economics, University of Foggia, Foggia, Italy

2 Department of Economics, Statistics and Finance, University of Calabria, Cosenza, Italy

3 Department of Law, University of Pisa, Pisa, Italy

4 Department of Finance, Faculty of Economics, Technical University of Ostrava, Ostrava, Czech Republic 
been considered. As far as this is concerned, we refer to Hommes and LeBaron (2018), for an exhaustive review about the use of agent-based models and nonlinear systems for describing the interaction among heterogeneous agents, and Massaro (2013), concerning the possibility of dealing with heterogeneous expectations in the so called Dynamic Stochastic General Equilibrium (DSGE) models.

Focusing on dynamic microeconomic models, the recent literature has shown how even a low degree of heterogeneity among agents with bounded rationality produces complex phenomena. Crucial along this line of research are the pioneering works of Bischi et al. $(1998,1999)$, in which the authors show that a lack of knowledge of the market (unknown market demand) and the interaction of two players with a little mismatch in parameters may generate non-synchronization of decisions and related complex phenomena.

On the same research strand, Fanti et al. (2015) show that in a duopoly market the interaction between owners and managers may destabilize the system even in the case in which agents are identical. Nevertheless, the agents' higher knowledge of the market, at least in the case of homogeneous players, seems able to avoid the onset of dynamics that do not converge to the equilibrium. Think for example of the classic article by Puu (1991) where the author considers players with complete knowledge of the market demand, having naive expectations on their competitors' next period actions and who use the best reply. In Puu's work, the non-convergence to the equilibrium is possible only if the duopolistic firms are very different from each other (if they have very different marginal costs).

Recently, part of the literature has focused on understanding how the interaction among groups of agents adopting heterogeneous strategies (Cavalli et al. 2015) and the possibility of switching between groups (Bischi et al. 2015) can generate non-trivial dynamic phenomena. The present work is directly connected to the articles of Bischi et al. $(1998,1999)$ and investigates whether the perfect knowledge of the economic mechanisms characterizing the market and the adoption of the best reply are capable of determining the loss (or achievement) of synchronization (in the short and long run) among the behaviors adopted by players, when applied to strategic contexts other than Puu (1991). In this context, we review the marketing model proposed by Farris et al. (2005) and further deepened by Bischi and Baiardi (2015a). We also consider an extension of the original model accounting for the dependence of the sales potential on customers' attraction (endogenous market size). We aim to show two main issues: (i) even in the presence of homogeneous agents, non-synchronized and complex behaviors can be observed due to the presence of multiple equilibria, and (ii) a low degree of heterogeneity may significantly affect the dynamics of the model among players due to existence of non-topological (Milnor) attractors. In particular, this last point shows that using the rhetoric of the representative agent to describe marketing dynamics may be misleading.

The paper is organized as follows. Section 2 shows the main features of the map, and the study of the dynamics along the diagonal is provided. Section 3 introduces the emergence of weak (Milnor) attractors. Section 4 is dedicated to the interpretation of various dynamic scenarios to highlight some dynamic properties of the model we consider. In Sect. 5 an extension of the model is formulated and 
possible achievements of synchronized trajectories are discussed. Section 6 concludes.

\section{The map and its symmetries}

We consider the discrete time dynamic map proposed in Bischi and Baiardi (2015a), which is based on the marketing competition model introduced by Farris et al. (2005). The model assumes $n$ profit maximizing firms selling homogeneous goods, whose decision variables are marketing efforts. Player $i$ sets marketing spending $x_{i}$ to obtain market share $s_{i}$ defined as

$$
s_{i}=\frac{A_{i}(t)}{A}
$$

where $A_{i}$ represents the attraction of consumers to $i$ and $A=\sum_{j=1}^{n} A_{j}(t)$ is the aggregate attraction. Following Farris et al. (2005) (see also Cooper and Nakanishi 1989), the specification $A_{i}=a_{i} x_{i}^{\beta_{i}}$ is assumed, where $a_{i} \geq 0$ represents the effectiveness of efforts expended by player $i$, while $\beta_{i}$ denotes the elasticity of attraction of the firm with respect to its marketing effort. Assuming unitary elasticities, the one period profit of firm $i$ is given by

$$
\Pi_{i}=B s_{i}-x_{i}=\frac{a_{i} x_{i}}{\sum_{j=1}^{n} a_{j} x_{j}}-x_{i}, \quad i=1, \cdots n
$$

where parameter $B$ represents the sales potential of the market, or market size. From the first-order condition, $i$ 's best response is obtained

$$
R_{i}\left(\sum_{j \neq i} a_{j} x_{j}\right)=\sqrt{B \frac{\sum_{j \neq i} a_{j} x_{j}}{a_{i}}}-\sum_{j \neq i} a_{j} x_{j}
$$

At a given time period, the effort $x_{i}$ is assumed to be determined by the convex combination between $i$ 's best response to their competitors' expected actions and its previous period decision. Assuming static expectations, the dynamic model is given by

$$
x_{i}^{\prime}=\left(1-\lambda_{i}\right) x_{i}+\lambda_{i} R_{i}\left(\sum_{j \neq i} a_{j} x_{j}\right), \quad i=1, \cdots n
$$

where ' represents the unit time advancement operator, while $\lambda_{i} \in(0,1)$ measures $i$ 's inertia or anchoring attitude. Such a particular kind of adjustment proposed in Farris et al. (2005) is known as adaptive adjustment towards the best reply (see also Puu 1991; Kopel 1996; Bischi et al. 2010). Map $T:(x, y) \rightarrow\left(x^{\prime}, y^{\prime}\right)$, considered in Bischi and Baiardi (2015a), is derived from (??) in the case of two firms in the new rescaled variables $x=a_{1} a_{2} x_{1}$ and $y=a_{1} a_{2} x_{2}$ : 
Fig. 1 Bifurcation diagram of $T_{\Delta}$ as $a$ varies.

$\lambda_{1}=\lambda_{2}=\lambda=0.98$

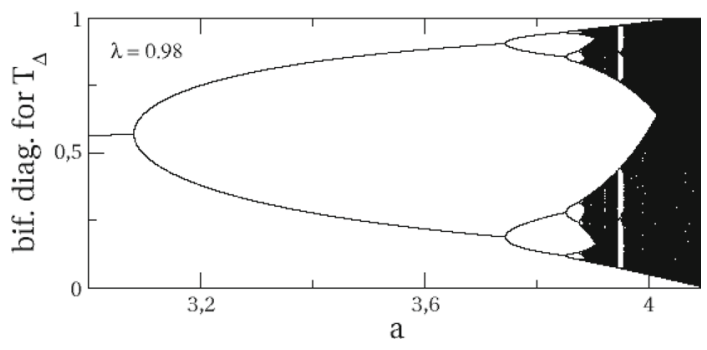

$$
T:\left\{\begin{array}{l}
x^{\prime}=\left(1-\lambda_{1}\right) x+\lambda_{1} a_{2}(\sqrt{y}-y) \\
y^{\prime}=\left(1-\lambda_{2}\right) y+\lambda_{2} a_{1}(\sqrt{x}-x) y^{\prime}=\left(1-\lambda_{2}\right) y+\lambda_{2} a_{1}(\sqrt{x}-x)
\end{array}\right.
$$

Feasible trajectories described by map $T$ are those characterized by nonnegative values of $x$ and $y$. To avoid events in which map $T$ generates negative values of the state variables after a finite number of iterations (then trajectory stops existing), we will assume $a_{i} \leq 4$ with $i=1,2$, such conditions ensuring the set $S=[0,1] \times[0,1]$ to be a trapping region (that is formed by points whose forward iterations remain included in $S$ ) and we will restrict the set of possible initial conditions to $S$.

\subsection{The symmetric case}

Local dynamic properties of map $T$ have been extensively discussed in Bischi and Baiardi (2015a), where existence conditions of fixed points of map $T$ and related local stability analysis have been provided. Moreover, the occurrence of global dynamic scenarios where coexisting attractors (often with complex structure) paired with complex structures of (often disconnected) basins of attractions has been discussed as well. Here we combine such local analysis with a remark concerning the restriction $T_{\Delta}$ of map $T$ to identical initial conditions, under the hypothesis of identical players. When $a_{1}=a_{2}=a$ and $\lambda_{1}=\lambda_{2}=\lambda$, map $T$ becomes symmetric. This means that map $T$ remains invariant if variables $x$ and $y$ are swapped, that is $T \circ W=W \circ T$ where $W:(x, y) \rightarrow(y, x)$. This implies that the diagonal $\Delta=\{(x, y): x=y\}$ is an invariant manifold, i.e., a trajectory starting at $\left(x_{0}, y_{0}\right) \in \Delta$ lies on $\Delta$ for all $t>0$, namely $\left(x_{t}, y_{t}\right) \in \Delta$ for all $t>0$.

Synchronized trajectories are governed by restriction $T_{\Delta}: \Delta \rightarrow \Delta$, where

$$
T_{\Delta}: x^{\prime}=f(x):=(1-\lambda) x+\lambda a(\sqrt{x}-x) .
$$

The following proposition summarizes basic dynamic properties of map $T$. We refer to Bischi and Baiardi (2015a) (Sect. 3) for the proof.

Proposition 1 Fixed points of map $T_{\Delta}$ given in (3) are $x_{0}^{*}=0$ and $x^{*}=(a /(a+1))^{2}$. Point $x_{0}^{*}$ is always unstable, while $x^{*}$ is locally asymptotically stable provided that $a<(4-\lambda) / \lambda$ is given. At $a=(4-\lambda) / \lambda$, point $x^{*}$ undergoes a flip bifurcation. 

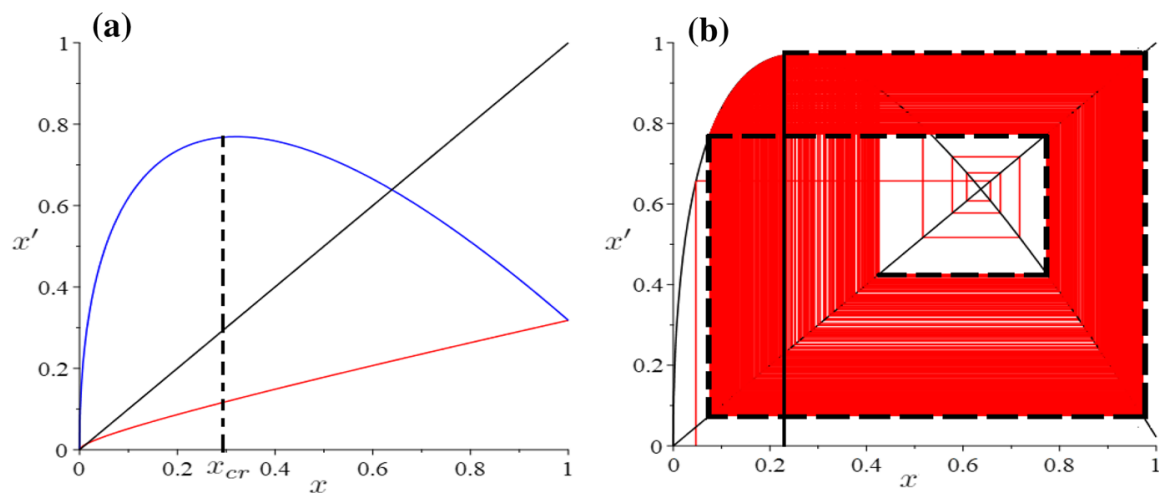

Fig. 2 a Different shapes of map $T_{\Delta}$ for $\lambda=0.682$. Monotonic case for $a=0.135$ (red curve) or unimodal case for $a=3.983$ (blue curve). The critical point $x_{c r}$ is evidenced by the dotted vertical line. $\mathbf{b}$ Parameters: $\lambda=0.98, a=3.994$. Chaotic regime for the system, trapped in the absorbing interval defined by dotted black lines $\left(x_{0}=0.047\right)$ (color figure online)

In Fig. 1 the bifurcation diagram of $T_{\Delta}$ is provided as parameter $a$ varies. It shows the period doubling cascade phenomenon occurring after the loss of stability of $x^{*}$ through flip bifurcation.

The following proposition provides further characterizations of map $T_{\Delta}$.

Proposition 2 Assuming $a \leq 4$, the map $T_{\Delta}$ given in (3) is concave. Moreover,

(a) if $a \in\left(0, \frac{2(1-\lambda)}{\lambda}\right]$, then $T_{\Delta}$ is monotonically increasing in $[0,1]$;

(b) if a $>\frac{2(1-\lambda)}{\lambda}$, the map has unimodal shape with critical point

$$
x_{c r}=\left(\frac{a \lambda}{2(a \lambda+\lambda-1)}\right)^{2}
$$

Proof (a) The proof is straightforward from the sign of both the first and second derivatives of (3) and the limits of $f$.

(b) The expression of $x_{c r}$ is obtained by solving the equation $f^{\prime}(x)=0$ and studying the space of parameters for which $x_{c r} \in[0,1]$.

Qualitative shapes of map $T_{\Delta}$ are shown in Panel (a) of Fig. 2 for smaller and greater values of parameter $a$ with respect to the threshold $2(1-\lambda) / \lambda$. The unimodality of $f(x)$ and the period doubling cascade described in Fig. 1 suggest that, given $\lambda$, for a sufficiently high value of $a$ dynamics may become irregular. Panel (b) in Fig. 2 shows this occurrence, where after a finite number of iterations, trajectory is trapped in an absorbing interval, bounded by the critical point (in the Julia-Fatou sense) $x_{c r}$ and its first four iterates. 


\section{The problems of synchronization and chaotic synchronization}

In Bischi and Baiardi (2015a) the authors complement the analysis provided in Farris et al. (2005) stressing the dynamic consequences of heterogeneities highlighting the way in which non-identical players, whose behaviors are characterized by different effectiveness and inertia parameters, influence the equilibrium points, their stability and bifurcations, as well as the global dynamic scenarios of the model.

A complementary approach has been adopted in Bischi and Baiardi (2015b), where the authors critically discuss the assumption, often considered in economic theory, of the existence of a representative agent, according to which identical or quasi identical players behave in identical or quasi identical ways (see, e.g., Kirman 1992; Aoki 1998; Bischi et al. 1999 for discussions on the topic). To this end, in the first part of that work, the authors account for three different dynamic adjustment processes belonging to the class of market share attraction models (see, e.g., Bell et al. 1975; Bonanno and Zeeman 1985; Carpenter et al. 1988; Bischi et al. 2000 or Farris et al. (2005)). The first adjustment consists in the model with the best reply and inertia introduced in Sect. 2. The second adjustment is proposed in Bischi et al. (2000), where agents are assumed to follow a profit-driven heuristic behavior with anchoring attitude. The third model is considered in Bischi and Kopel (2003) and derived assuming agents to follow the so called gradient adjustment mechanism (see Bischi and Naimzada 2000 and the reference therein). Remarkably, when such $n$-dimensional dynamic models are reduced to one-dimensional maps under the assumption of identical players with the same initial condition, the role of the numerosity $n$, taken as a bifurcation parameter, has been shown to determine different and sometimes opposed behaviors of the related synchronized dynamics. Then, in the second part of Bischi and Baiardi (2015b), the authors deepen the analysis of the marketing model proposed in Bischi et al. (2000) if two competing agents are assumed. The problem of synchronization achievement is addressed by studying the stability property of the attractor placed along the (invariant) synchronization manifold $\Delta$. This corresponds to the search for conditions for which $\lim _{t \rightarrow \infty}\left\|x_{t}-y_{t}\right\|=0$ holds, given generic initial conditions $\left(x_{0}, y_{0}\right)$. From an interpretative point of view, this means that, when synchronization patterns are reached in the long run, behavioral homogeneity among players is achieved, even when initial configurations of the population are characterized by behavioral heterogeneity.

Along this line we ask whether synchronized or quasi-synchronized dynamics can be described by map $T$, given in (2), in the non-generic situation in which identical or quasi-identical agents are characterized by different initial conditions. Again, this means establishing whether identical (resp. similar) competitors starting from different initial conditions will behave homogeneously (res. similarly) in the long run, so that the asymptotic behavior of the system is governed by the one-dimensional restriction $T_{\Delta}$ (resp. takes place in a neighborhood of $\Delta$ ). We present here a brief overview of results that will be used to address this problem (see to this purpose Alexander et al. 1992; Ashwin et al. 1996; Buescu 2012; Venkataramani et al. 1996; Bischi et al. 1998; Bischi and Gardini 2000 and Bischi and Cerboni Baiardi (2017)).

Let us start considering the case of identical players, assuming that $a_{1}=a_{2}=a$ and $\lambda_{1}=\lambda_{2}=\lambda$ hold, and let $A_{s} \subset \Delta$ be a closed invariant set for $T_{\Delta}$ such that $T\left(A_{s}\right)=A_{s}$. The analysis of the transverse stability of $A_{s}$ will reveal whether it is 
an attractor also for $T$ and can be performed by considering the Jacobian matrix of $T$ and restricted to $\Delta$ :

$$
J(x, x)=\left(\begin{array}{cc}
l(x) & m(x) \\
m(x) & l(x)
\end{array}\right)
$$

The eigenvalues associated with a generic point $(x, x) \in \Delta$ are

$$
\nu_{\|}(x)=l(x)+m(x)=1-\lambda+\lambda a\left(\frac{1}{2 \sqrt{x}}-1\right),
$$

with eigenvector $(1,1)$ (parallel to $\Delta$ ) and

$$
v_{\perp}(x)=l(x)-m(x)=1-\lambda-\lambda a\left(\frac{1}{2 \sqrt{x}}-1\right)
$$

with eigenvector $(1,-1)$ (orthogonal to $\Delta$ ). When the invariant set $A_{s}$ embedded along $\Delta$ is a $k$-cycle of the form $C_{k}=\left\{\left(x_{1}, x_{1}\right), \ldots,\left(x_{k}, x_{k}\right)\right\}$, where $k \in \mathbb{N} \backslash\{0\}$, its stability is measured by means of the product of the eigenvalues computed at each point $\left(x_{i}, x_{i}\right) \in C_{k}$, with $i=1, \ldots, k$, namely

$$
v_{\|}^{(k)}=\prod_{i=1}^{k}\left(l\left(x_{i}\right)+m\left(x_{i}\right)\right)
$$

and

$$
v_{\perp}^{(k)}=\prod_{i=1}^{k}\left(l\left(x_{i}\right)-m\left(x_{i}\right)\right)
$$

In detail, since $v_{\|}=f^{\prime}(x)$ for the one-dimensional map defined in (3), it follows that $v_{\|}^{(k)}$ is the eigenvalue of the $k$-cycle with eigenvector along the diagonal $\Delta$, while $v_{\perp}^{(k)}$ is the eigenvalue of the $k$-cycle along the direction normal to $\Delta$. Then, the stability of $C_{k}$ along $\Delta$ is guaranteed by the condition $\left|v_{\|}^{(k)}\right|<1$, while the transverse stability of the same cycle is guaranteed by the condition $\left|v_{\perp}^{(k)}\right|<1$. Then, when the two previous conditions are matched, it can be concluded that a $C_{k}$ is locally asymptotically stable.

Differently, if $A_{s}$ is a chaotic attractor, the problem becomes more interesting and the phenomenon of chaos synchronization may be observed (see, e.g., Fujisaka and Yamada 1983; Pecora and Carroll 1990; Lai et al. 1996). Such an occurrence may be paired with a weaker transverse stability property of $A_{s}$ than local asymptotic stability. In this case, Milnor attractors that are not stable in Lyapunov sense may appear (see Milnor 1985). In order to understand the meaning of this phenomena, we recall for convenience some well-known definitions. Let $B\left(A_{s}\right)$ be the basin of attraction of $A_{s}$, namely the set of points whose $\omega$-limit set belongs to $A_{s}$. The (strongest) notions of stability are provided in the following definition.

Definition 3 The closed invariant set $A_{s}$ is an asymptotically stable attractor (or topological attractor) if it is Lyapunov stable, i.e., for every neighborhood $U$ of $A_{s}$ there 
exists a neighborhood $V$ of $A_{s}, V \subset U$, such that $T^{t}(V) \subset U$ for every $t \geq 0$, and $B\left(A_{S}\right)$ contains a neighborhood of $A_{s}$.

According to Definition 3, when $A_{s}$ is a topological attractor, then a neighborhood (with positive Lebesgue measure) surrounding $A_{s}$ and included in $B\left(A_{s}\right)$ exists. This implies that $B\left(A_{s}\right)$ is characterized by a positive Lebesgue measure as well. Conversely, it is only necessary for $A_{S}$ to be locally asymptotically stable to have $B\left(A_{S}\right)$ with positive Lebesgue measure.

Definition 4 A closed invariant set $A$ is said to be a weak attractor in Milnor sense (or simply Milnor attractor) if its basin of attraction $B\left(A_{s}\right)$ has positive Lebesgue measure.

Then, a topological attractor is also a Milnor attractor, whereas the converse is not always true. Roughly speaking, a Milnor attractor may be unstable (in Lyapunov sense) while attracting a set of points with positive measure.

In the present context, the chaotic set $A_{s} \subset \Delta$ may turn from an asymptotically stable set to a weak or non-topological Milnor attractor, because it includes infinitely many periodic orbits, dense in $A_{s}$, which are unstable in the direction along $\Delta$. Such cycles may be characterized by different degrees of transverse attractiveness and it may happen that some of them are repelling in the direction orthogonal to $\Delta$, while some others are attractive along the same direction. Then, the presence of at least one transversely repelling cycle will prevent $B\left(A_{S}\right)$ from including a neighborhood of $A_{s}$, since any initial condition placed out of $\Delta$ and sufficiently close to the cycle will be iterated far from $A_{s}$. This, however, does not prevent $B\left(A_{s}\right)$ from having positive measure. The transverse attractiveness of a trajectory $\left\{x_{t}=T^{t}\left(x_{0}\right), t \geq 0\right\}$ embedded on $A_{s}$ can be evaluated by means of the associated transverse Lyapunov exponent:

$$
\Lambda_{\perp}=\lim _{T \rightarrow \infty} \frac{1}{T} \sum_{t=0}^{T} \log \left|v_{\perp}\left(x_{t}\right)\right|
$$

Clearly, if $\left(x_{0}, x_{0}\right)$ is a point of a $k$-cycle, or belongs to the stable set of a $k$-cycle, then $\Lambda_{\perp}=\ln \left|v_{\perp}^{k}\right|$ and the cycle is transversely stable when $\Lambda_{\perp}<0$. Differently, if $\left(x_{0}, x_{0}\right)$ belongs to a generic aperiodic trajectory embedded inside the chaotic set $A_{s}$, then $\Lambda_{\perp}$ is the so called natural ${ }^{1}$ transverse Lyapunov exponent $\Lambda_{\perp}^{\text {nat }}$, which provides the measure of the average transverse attractiveness of $A_{s}$. It accounts for the local average behavior of trajectories in a neighborhood of $A_{s}$ and allows to identify bifurcations such as the riddling bifurcation or the blowout bifurcation. Since infinite cycles, all unstable along $\Delta$, are embedded in a chaotic attractor $A_{s}$, a spectrum of transverse Lyapunov exponents can be defined:

$$
\Lambda_{\perp}^{\min } \leq \ldots \leq \Lambda_{\perp}^{\text {nat }} \leq \ldots \leq \Lambda_{\perp}^{\max }
$$

where inequalities remark the property of $\Lambda_{\perp}^{\text {nat }}$ as the average between the transversely repelling and transversely attracting cycles. In particular, (i) if $\Lambda_{\perp}^{\max }<0$, then all the

\footnotetext{
1 The term natural refers to the Lyapunov exponent associated to the Sinai-Bowen-Ruelle measure, computed for a typical trajectory taken in the chaotic attractor $A_{s}$ (see, e.g., Buescu 2012.
} 
cycles embedded in $A_{s}$ are transversely attracting and $A_{s}$ is asymptotically stable for map $T$; (ii) if $\Lambda_{\perp}^{\max }>0$ while $\Lambda_{\perp}^{\text {nat }}<0$, some cycles (together with their preimages) nested in the chaotic set $A_{s}$ exist, whose transverse Lyapunov exponents are positive, so that they are transversely repelling. Then $A_{s}$ is no longer Lyapunov stable. However, since the majority of the trajectories on $A_{s}$ are transversely attractive (which follows from the average transverse attractiveness of $A_{s}$ ), a set of points with positive measure belongs to $B\left(A_{s}\right)$ and $A_{s}$ reduces to a non-topological Milnor attractor.

The transition from asymptotic stability to stability only in Milnor sense, recognized through a change in the sign of $\Lambda_{\perp}^{\max }$ from negative to positive, is the so-called riddling bifurcation (or bubbling bifurcation). If also $\Lambda_{\perp}^{\text {nat }}$ becomes positive, the transversely unstable periodic orbits embedded into $A_{s}$ have a greater weight as compared to the stable ones. Then, $A_{s}$ is no longer a Milnor attractor, since it attracts a set of points of zero measure, and it becomes a chaotic saddle. We refer to Buescu (2012) for deeper discussions. The transition of $A_{s}$ from a non-topological Milnor attractor to a chaotic saddle occurs through the so called blowout bifurcation.

We dedicate a final remark in this section to highlight that riddling bifurcation may have different consequences on global dynamic scenarios. Indeed, even if the occurrence of riddling bifurcation is detected studying the sign of the natural Lyapunov exponent, its effect depends by global dynamic properties of the system. In detail, after the riddling bifurcation, trajectories that are locally repelled near the local unstable manifolds of the transversely repelling cycles may be redirected on $\Delta$. This event is referred to as local riddling in the literature (see Venkataramani et al. 1996; Zimin et al. 2003), where such trajectories are characterized by few bursts away from $\Delta$ before synchronizing on it, giving rise to the so-called on-off intermittency phenomenon (see Ashwin et al. 1996; Venkataramani et al. 1996). Differently, trajectories that are locally repelled away from $A_{s}$ may reach another attractor, being included in its basin. This event is referred to as local riddling in the literature. Global riddling is also paired to emergence of so-called riddled basins (see Alexander et al. 1992), where each subset of $B\left(A_{S}\right)$ includes points of the basin of the other attractor. Similarly, effects of blowout bifurcations are affected by global dynamic features of the system far from the invariant manifold $\Delta$. In detail, the trajectories starting close to the chaotic saddle may be attracted by another attracting set far from the diagonal or, alternatively, they remain enclosed inside a two-dimensional compact set surrounding $A_{s}$, giving rise to an endless on-off intermittency. A method based on critical lines has been proposed in Bischi and Cerboni Baiardi (2017) to characterize the dynamic consequences of riddling and blowout bifurcations.

\section{Synchronized and quasi-synchronized dynamic patterns and related stability notions}

In this section we provide some dynamic scenarios described by map $T$ given in (2) and we discuss them in the light of the arguments presented in Sect. 3. We place the analysis in the symmetric case and set $\lambda=0.98$ in simulations. It is worth noting that both parameters $a$ and $\lambda$ influence transverse stability of attractors embedded along $\Delta$. This follows from the expression of the eigenvalue $v_{\perp}$ provided in (6), which 

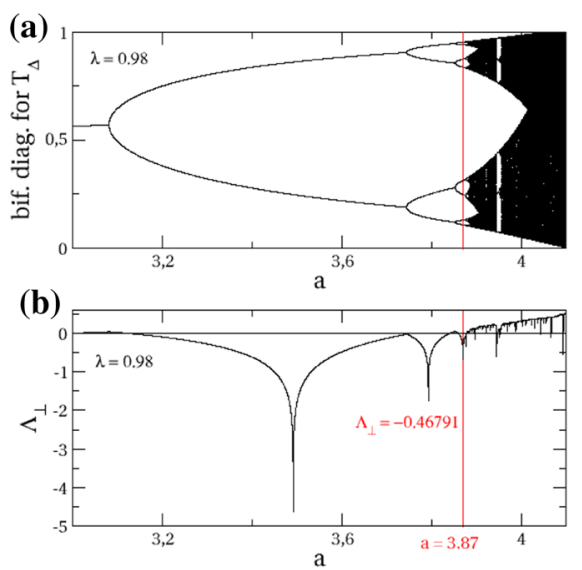

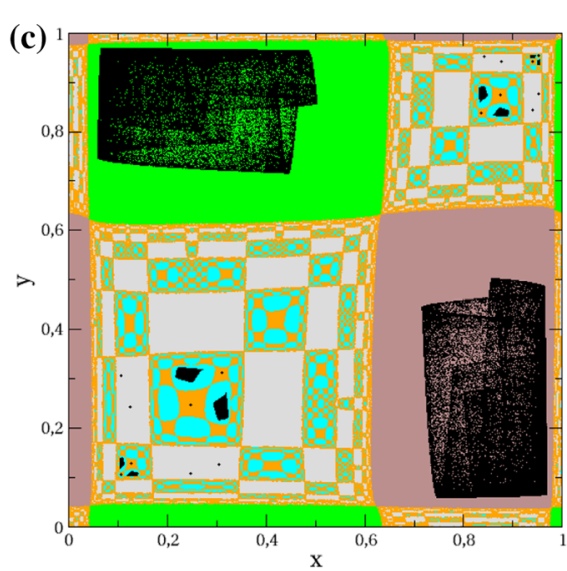

Fig. $3 \Lambda_{\perp}=-0.46791, \lambda=0.98, a_{1}=a_{2}=3.87$. a Bifurcation diagram with respect to the parameter $a$ highlights the occurrence of a 8-cycle (see the vertical line in red). b Associated Lyapunov exponent diagram. $\mathbf{c}$ Basins of attraction in the plane $(x, y)$ (color figure online)

depends on both parameters $\lambda$ and $a$. However, at the same time, parameters $a$ and $\lambda$ influence the dynamics along $\Delta$, which follows from the dependence of the eigenvalue $v_{\|}$provided in (5) by both $a$ and $\lambda$ (see, e.g., the bifurcation diagram given in Fig. 1 obtained by varying parameter $a$ ). As a consequence, variations of even a single parameter do not preserve chaotic dynamics along $\Delta$. Hence, numerical computations of $\Lambda_{\perp}$ do not necessarily represent the natural transverse Lyapunov exponent $\Lambda_{\perp}^{\text {nat }}$ because when a parameter varies within a periodic window of the bifurcation diagram, the trajectory is captured by a stable cycle. In this situation, $\Lambda_{\perp}$ only represents the Lyapunov exponent of that cycle. However, when the trajectory is captured by a highperiod cycle, the trajectory visits an important portion of the set $A_{s}$ and its transverse Lyapunov exponent can be considered an approximation of the natural one. We then adopt the methods considered in Bischi and Cerboni Baiardi (2017) in studying the dynamic system proposed in Bischi and Lamantia (2002a) or Bischi and Lamantia (2002b). The sequence of the dynamic scenarios provided in this section is obtained by varying parameter $a$ and simulations are ordered to illustrate the sequence of (local and global) riddling and bubbling global bifurcations in the way they are presented in Sect. 3 (namely as zero moves from the right to the left in the Lyapunov exponent's spectrum).

The first scenario is presented in Fig. 3. In Panel (a) the bifurcation diagram of map $T_{\Delta}$ is paired with Panel (b) showing transverse Lyapunov exponents of trajectories nested in $\Delta$ as $a$ varies. (The red line highlights the selected value of $a$ for the simulation in Panel (c).) In detail, at $a=3.87$, the bifurcation diagram reveals the presence of a period 8-cycle along $\Delta$, which is locally asymptotically stable. In this case, the associate transverse Lyapunov exponent is negative $\left(\Lambda_{\perp}=-0.46791\right)$. It is interesting to note that, differently from many contributions involving duopoly dynamics with symmetric maps (such as Bischi et al. 1999), where only attractors 


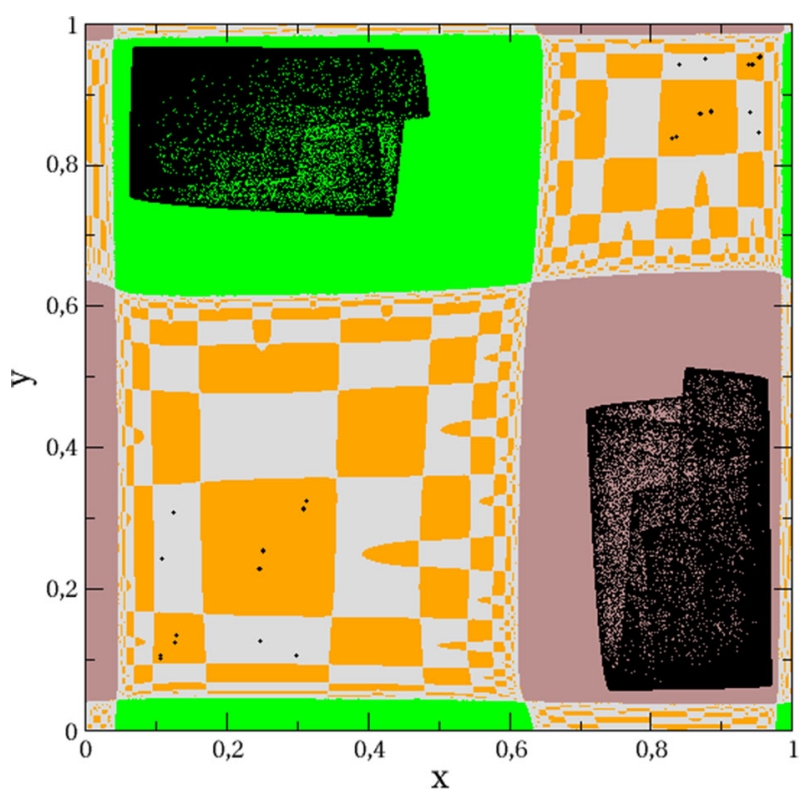

Fig. $4 \lambda=0.98, a_{1}=a_{2}+0.01, a_{2}=3.87$

along the diagonal exist, coexistence of multiple attractors is here observed. ${ }^{2}$ Those are two attractors formed by an even number of pieces symmetrical with respect to the diagonal (a 8-cycle and a chaotic attractor with 8 pieces) and two other chaotic attractors in a symmetric position (see Panel (c) in Fig. 3). The different colors applied in Panel (c) identify the different basins of attractions of the attractors described above.

Since the 8-cycle along $\Delta$ is locally asymptotically stable, the introduction of small heterogeneities among agents through slight changes between parameters $a_{1}$ and $a_{2}$ implies that the same period 8-cycle undergoes smooth changes with respect to the symmetric case, as shown in Fig. 4. In this case, the description of the model with a unique representative player instead of two similar competitors (with initial conditions close to the attractor) provides a good approximation of the marketing dynamics. Of course, besides the smooth modification of the 8-cycle originally included in $\Delta$, the mismatch between parameters $a_{1}$ and $a_{2}$ causes both local and global bifurcations, as shown in Fig. 4, where the cycle along the diagonal has been transformed into a 16-cycle not belonging to $\Delta$ via a flip bifurcation, while the contact between 8 pieces chaotic attractor and the boundaries of its basin has destroyed the chaotic attractor.

Going back again to the symmetric case, the second scenario is presented in Fig. 5, obtained at a higher value of the parameter $a$, now fixed at $a=3.9449$. Such variation causes a crucial change in the scenario, where $A_{s}$ is the unique attrac-

\footnotetext{
${ }^{2}$ For sake of completeness, the occurrence of multistability together with synchronized trajectories has been investigated in several contributions. See, for example, Maistrenko et al. (1997, 1998b), Popovych et al. (2000, 2001), Kapitaniak (2001); Neumann et al. (2003).
} 

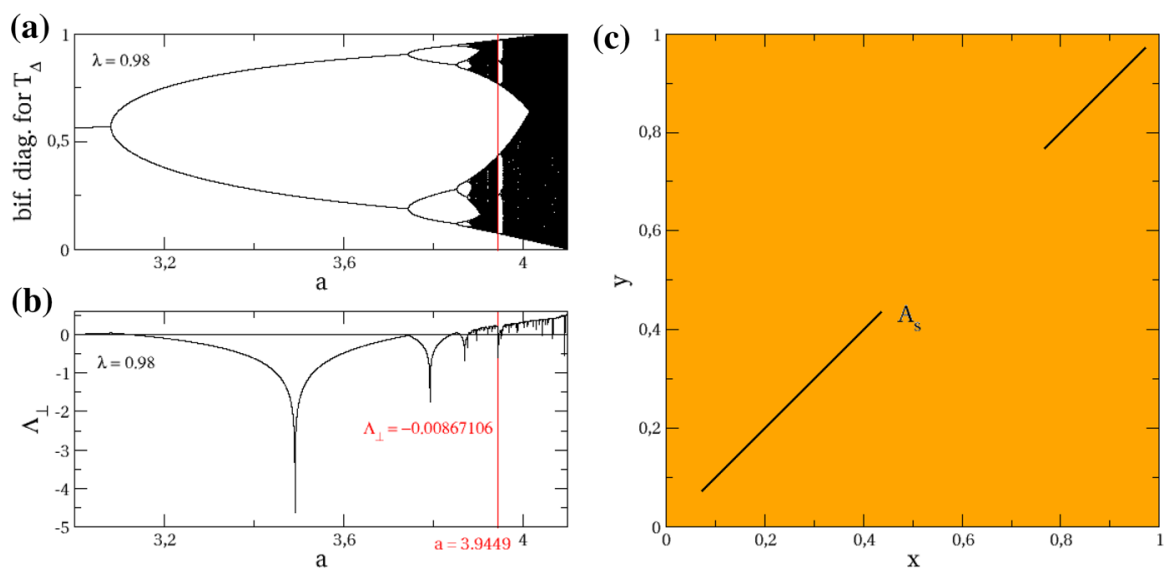

Fig. $5 \Lambda_{\perp}^{\text {nat }}=-0.00867106, \lambda=0.98, a=3.9449$

tor of the system (see Panel (c)). As shown in Fig. 5 Panel (a), the restricted map $T_{\Delta}$ exhibits a chaotic attractor (or a high-periodic cycle). At the same time, the transverse Lyapunov exponent of the trajectory observed in simulation is negative (precisely, $\Lambda_{\perp}^{\text {nat }}=-0.00867106$, see Panel (b) in Fig. 5). Moreover, the transverse Lyapunov exponent associated to the 2-cycle $C_{2}$ embedded in $A_{s}{ }^{3}$ results $\Lambda_{\perp}\left(C_{2}\right)=0.316569>0$. This shows that $A_{s}$ is a non-topological Milnor attractor and attracts a set of points with positive (Lebesgue) measure. We stress that, in Panel (c), the orange points represent the closure of the basin $B\left(A_{s}\right)$ of $A_{s}$, namely the numerical basin of $A_{s}$ including infinitely many repelling cycles existing outside $\Delta$, the presence of which can be argued also from the simulations presented both in Figs. 3 and 4.

Figure 6 Panel (a) shows how the convergence to attractor $A_{s}$ from initial conditions close to the diagonal can be very long with phases of on-off intermittency. This is a consequence of the fact that $A_{s}$ is a non-topological Milnor attractor. Indeed, the onoff phenomenon can be explained by the presence of transversely repelling cycles nested in $A_{s}$ and characterized by positive transverse Lyapunov exponents. Their presence indeed has the effect of iterating far from $A_{s}$ trajectories approaching it. Such trajectories, however, will be reinjected in to $A_{s}$ in the long run, being attracted by transversely attracting cycles of $A_{s}$. We further mention that the riddling bifurcation which caused the transition of $A_{s}$ from a locally asymptotically stable attractor to a non-topological Milnor attractor is of local type. In Figure 6 Panel (b), the scenario

3 The 2-cycle $C_{2}$ embedded in $A_{s}$ is $C_{2}=\left\{\left(x_{1}, x_{1}\right),\left(x_{2}, x_{2}\right)\right\}$, where

$$
\begin{aligned}
& x_{1}=\left(\frac{\lambda a}{2(\lambda+\lambda a-2)}\left(1-\sqrt{1-\frac{4}{\lambda+\lambda a}}\right)\right)^{2} \\
& x_{2}=\left(\frac{\lambda a}{2(\lambda+\lambda a-2)}\left(1+\sqrt{1-\frac{4}{\lambda+\lambda a}}\right)\right)^{2}
\end{aligned}
$$



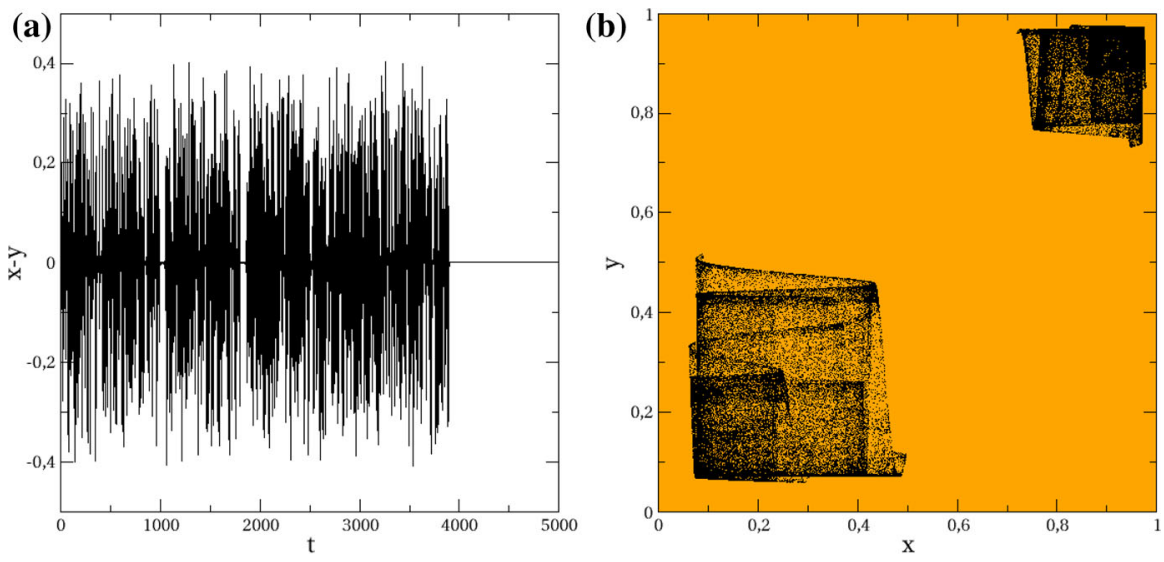

Fig. 6 a Time series of $x-y$ describing the on-off intermittency phenomenon, where $\Lambda_{\perp}^{\text {nat }}=-0.00867106$, $\lambda=0.98, a=3.9449$. b Change of the attractor when a mismatch between $a_{1}$ and $a_{2}$ is introduced; $\lambda=0.98, a_{1}=a_{2}+0.01, a_{2}=3.9449$

highlighted in Figure 5, obtained in the symmetric case, is varied introducing a small mismatch between parameters $a_{1}$ and $a_{2}$. This causes the destruction of the invariant manifold as well as an abrupt change of the qualitative behavior of the asymptotic dynamics, which now appears to be a two-dimensional chaotic set confined within an extended absorbing area. This outcome can be explained as follows: after the symmetry breaking, synchronization no longer occurs and the action of transversely unstable periodic cycles originally embedded in $A_{s}$ now determines an alternate sequence of departures and approaches of the trajectory from the invariant manifold, filling up the minimal absorbing area surrounding $A_{s}$. This gives rise to endless on-off intermittency where bursts never stop. In this situation, the use of the representative agent's rhetoric to describe marketing dynamics is certainly misleading.

Let us go back again to the symmetric case and let us fix $a=3.89672$. As in the previous scenario, the natural Lyapunov exponent is supposed to be negative and well approximated by computation of $\Lambda_{\perp}=-0.0363289$. The related dynamic scenario is sketched in Fig. 7 Panel (c). Also in this case, $A_{s}$ is conjectured to be a non-topological Milnor attractor. This supposition is justified by looking at the rarefied structure of $A_{s}$ 's basin represented by orange points. The basin $B\left(A_{s}\right)$ indeed looks intermingled with the basin of the 4-pieces chaotic attractor placed out of $\Delta$ having its basin represented by gray points, thus revealing the presence of riddled basins. Such an outcome is coherent with the non-topological nature of $A_{s}$, whose transversely unstable orbits are characterized by unstable manifolds belonging to the basin of an attractor different from $A_{s}$. Even if numerical simulations cannot reveal the very fine structure of $B\left(A_{s}\right)$ near $\Delta$, infinitely many (grey) tongues of the basin of stable chaotic set placed out of $\Delta$ can be thought as picking out from $A_{s}$, including unstable manifolds of transversely repelling cycles. The riddling is of global type in this case.

Starting from the same situation represented in the previous simulation, by further increasing the value of $a$ up to $a=3.9$ the natural transverse Lyapunov exponent becomes positive, being computed $\Lambda_{\perp}=0.11128$ along a high-period cycle (see 

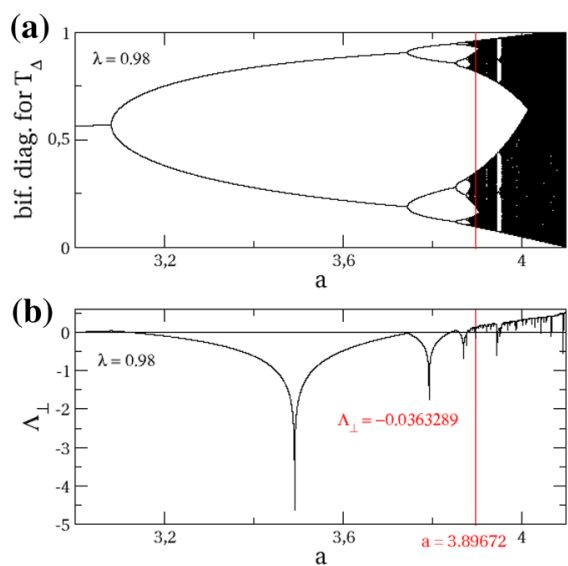

Fig. $7 \Lambda_{\perp}^{\text {nat }}=-0.0363289, \lambda=0.98, a=3.89672$

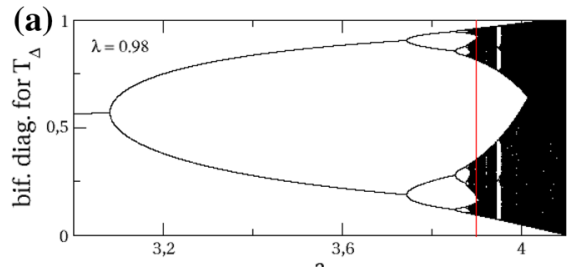

(b)

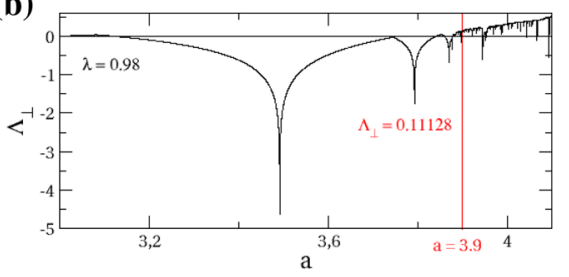

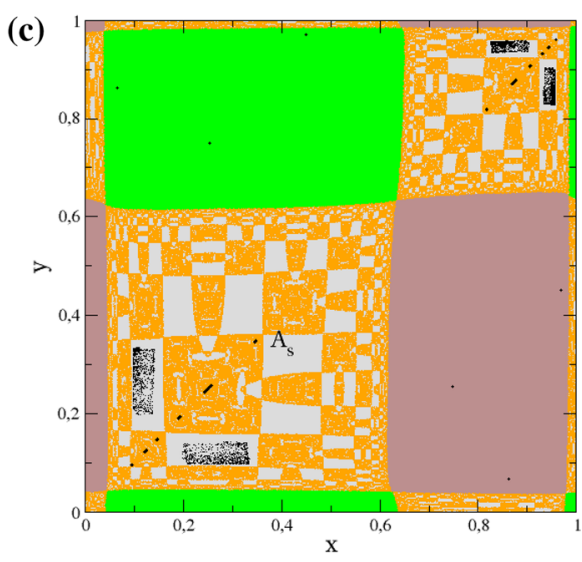

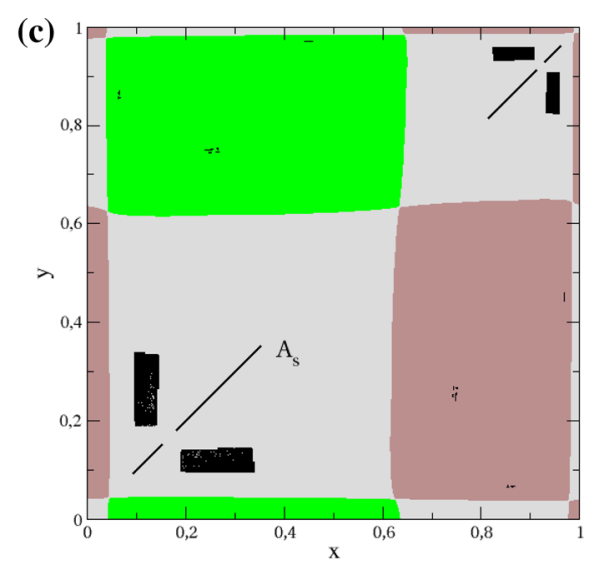

Fig. $8 \Lambda_{\perp}^{\text {nat }}=0.11128, \lambda=0.98, a=3.9$

Panels (a) and (b) in Fig. 8). The rise of $a$ then determines blowout bifurcation and $A_{s}$ turns into a chaotic saddle. The consequence of this global bifurcation on global dynamics is to prevent synchronization from being achieved for most of the trajectories that do not start along the diagonal, which are no longer attracted by $A_{s}$ neither confined in some area surrounding $A_{s}$ (as highlighted in Fig. 8 Panel (c)). Indeed, even if periodic cycles that are transversely attractive may still exist along $\Delta$, the initial conditions whose $\omega$-limit set belong to $\Delta$ is a set of zero measure.

\subsection{Absorbing areas and dynamic consequences of riddling and blowout}

In the previous sections we mentioned that riddling and blowout bifurcations may have, each on its own, different consequences on global dynamic scenarios. We stress that such different outcomes from each bifurcation are locally equivalent along $\Delta$ and can- 
(a)

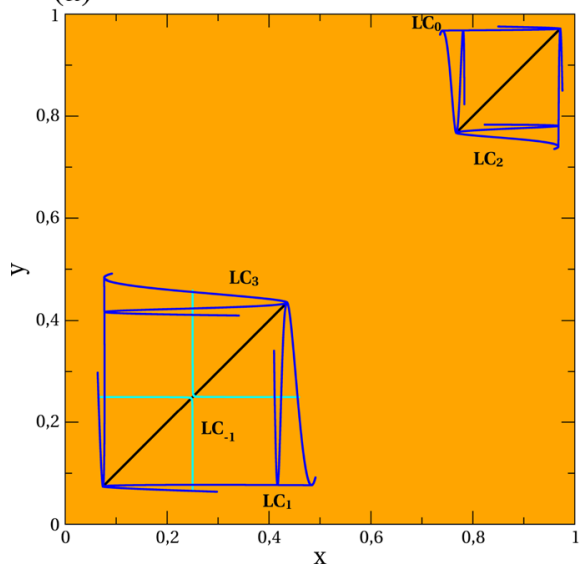

(b)

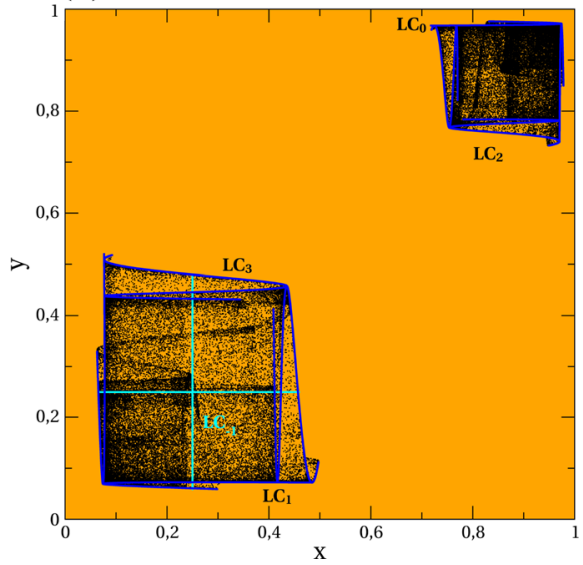

Fig. 9 Blue lines are the iterations $L C_{k}=T^{k}(L C)$ of the critical line $L C$, with $k=0, \ldots, 3$, representing boundaries of an absorbing area enclosing the chaotic attractor $A_{s}$ embedded along the diagonal. Light blue segments represent generating segments $L C_{-1}$. Parameters in Panel (a) and (b) are as in Figs. 5 and 6 , respectively (color figure online)

not be distinguished on the basis of the study of the spectrum of Lyapunov exponents. This problem is addressed in Bischi and Cerboni Baiardi (2017), where the authors exploit properties of (minimal) absorbing areas associated to non-invertible maps to explain occurrences of different global scenarios, observed after both riddling and blowout. We remark that this idea is originally suggested in Bischi and Gardini (1998) and in Maistrenko et al. (1998a) and considered also in Bischi et al. (1998) and Bischi and Gardini (2000). This concept is based on the crucial property of absorbing areas that are regions of the plane where trajectories entering such areas will never escape out of them. Indeed, absorbing areas are, in the simplest case, made up of the finite union of segments of the so called critical lines and their rank- $k$ images, having peculiar folding actions on portions of the plane inherited by the non-invertible structure of the map. We refer to Abraham et al. (1997); Gumowski and Mira (1980) and Mira et al. (1996) for more details. Then, different consequences of riddling bifurcation can be understood when an absorbing area surrounds a non-topological Milnor attractor. Local riddling occurs when locally repelled trajectories are folded back towards the attractor by boundaries of the absorbing area. Differently, when the absorbing area includes a portion of the basin of a second attractor (including it), trajectories that locally repelled away from the synchronization manifold may belong to the basin of the second attractor and move towards it in the long run. In the present context, the former occurrence is related to the simulation provided in Fig. 5. Boundaries of the minimal absorbing area enclose bursts occurring after synchronization is achieved and are represented in Fig. 9 Panel (a). These boundaries are made up of the critical line $L C$ and its iterations $T^{k}(L C)$, with $k=1,2,3$. In turn, the set $L C$ is obtained by one iteration through $T$ of the so-called generating segments $L C_{-1}$, given by the union of two branches included in the set of points where the Jacobian matrix associated to map $T$ vanishes. Also, after the introduction of the small mismatch between parameters $a_{1}$ 


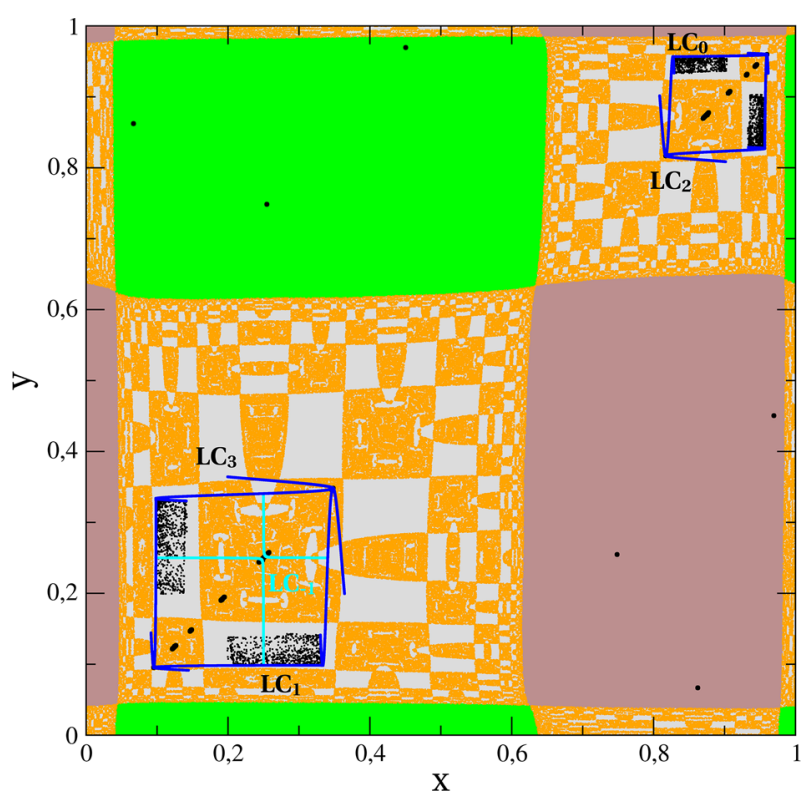

Fig. 10 Blue lines are the iterations $L C_{k}=T^{k}(L C)$ of the critical line $L C$, with $k=0, \ldots, 3$, representing boundaries of an absorbing area enclosing the chaotic attractor $A_{s}$ embedded along the diagonal. Light blue segments represent generating segments $L C_{-1}$. A second chaotic attractor included in the absorbing area and placed out of $\Delta$ is present. Parameters are as in Fig. 7 (color figure online)

and $a_{2}$ and the following symmetry breaking, the same slightly modified absorbing area encloses bursts along the endless on-off intermittency and provides the boundaries for the chaotic set shown in Fig. 6 Panel (b). This is shown in Figs. 9 Panel (b), 10 .

The latter occurrence is related to the simulation provided in Fig. 7. The absorbing area surrounds $A_{s}$ as well as the 4-piece chaotic attractor placed out of $\Delta$ together with portions of both their basins. Hence, locally repelled trajectories from $A_{s}$ can move towards the second attractor, thus causing global riddling and determining the presence of riddled basins. The absorbing area in this case is represented in Fig. 13.

\section{Endogenous market size}

In the formulation of the model discussed in the previous sections, a fixed market size is assumed. Therefore, the players compete in order to increase their own market share. Nevertheless, strong marketing efforts may create positive spillovers for the entire industry by attracting new consumers from other sectors. In particular, even if aimed at contrasting rivals, marketing expenditures allocated to increase the firms attraction may create positive effects on the entire market, which expands. See, e.g., Karnani (1985), Basuroy and Nguyen (1998) or Federgruen and Yang (2009) for contributions where aggregate sales have been considered as functions of the aggregate attraction. For this reason, we consider here an extension of the model discussed in Bischi and 
Baiardi (2015a), according to which the market size $B$ depends on $A$ as

$$
B(A)=B_{0} A^{\theta}, 0 \leq \theta<1 .
$$

where $B_{0}>0$ and parameter $\theta$ represents the elasticity of the market size with respect to the aggregate $A^{4}$. Equation (9) entails that the marketing effort made by the single player has two different effects: it affects the player's market share and, at the same time, it modifies the market size $B$. However, agents neglect to account for the dependence of $B$ on their own individual actions. In other words, each agent observes the present value of $B$, but considers this value as given in determining the best reply. Therefore, assuming that player have static expectations, best responses reads as

$$
\tilde{R}_{i}\left(\sum_{j \neq i} a_{j} x_{j} ; B(A)\right):=\sqrt{B_{0} A^{\theta} \frac{\sum_{j \neq i} a_{j} x_{j}(t)}{a_{i}}}-\sum_{j \neq i} a_{j} x_{j}(t), \quad i=1, \cdots, n
$$

Adaptive adjustments towards the best reply lead to the following dynamic system

$$
x_{i}^{\prime}=\left(1-\lambda_{i}\right) x_{i}+\lambda_{i} \tilde{R}_{i}\left(\sum_{j \neq i} a_{j} x_{j} ; B(A)\right), i=1, \cdots n
$$

where, again, parameters $\lambda_{i} \in(0,1)$ with $i=1, \cdots n$.

Remark 5 We remark that the model here considered boils down to the model considered in Bischi and Baiardi (2015a) when $\theta=0$.

Setting $B_{0}=1$, the market size results $B=\left(a_{1} x_{1}+a_{2} x_{2}\right)^{\theta}$ and the dynamics in the rescaled variables $x=a_{1} a_{2} x_{1}$ and $y=a_{1} a_{2} x_{2}$ is described by the two dimensional $\operatorname{map} \tilde{T}:(x, y) \rightarrow\left(x^{\prime}, y^{\prime}\right)$ :

$$
\tilde{T}:\left\{\begin{array}{l}
x^{\prime}=\left(1-\lambda_{1}\right) x+\lambda_{1} a_{2}\left(\sqrt{y\left(\frac{x}{a_{2}}+\frac{y}{a_{1}}\right)^{\theta}}-y\right) \\
y^{\prime}=\left(1-\lambda_{2}\right) y+\lambda_{2} a_{1}\left(\sqrt{x\left(\frac{x}{a_{2}}+\frac{y}{a_{1}}\right)^{\theta}}-x\right)
\end{array}\right.
$$

Differently from map $T$, whose dynamics are trapped in the region $S=[0,1] \times[0,1]$ when $a_{i} \leq 4$ is assumed, for map $\widetilde{T}$ it is difficult to analytically determine a condition on the parameters such that all the trajectories live (at each iteration) in $S$. Regarding the study of the region in which the dynamics of map $\tilde{T}$ takes place for each $t \geq 0$, we begin by noting that, without imposing any restrictions on the parameters, trajectories starting on the axes are either (i) reinjected into the interior part of the nonnegative

\footnotetext{
4 The functional form considered in (9) has been introduced in Karnani (1985).
} 
Fig. 11 Critical curves $L C=L C^{(a)} \cup L C^{(b)}$ (black) which separates regions $Z_{4}, Z_{2}$ and $Z_{0}$ whose points have 4,2 and no rank-1 preimages, respectively. Boundary $H$ (red) surrounds feasible region $F$

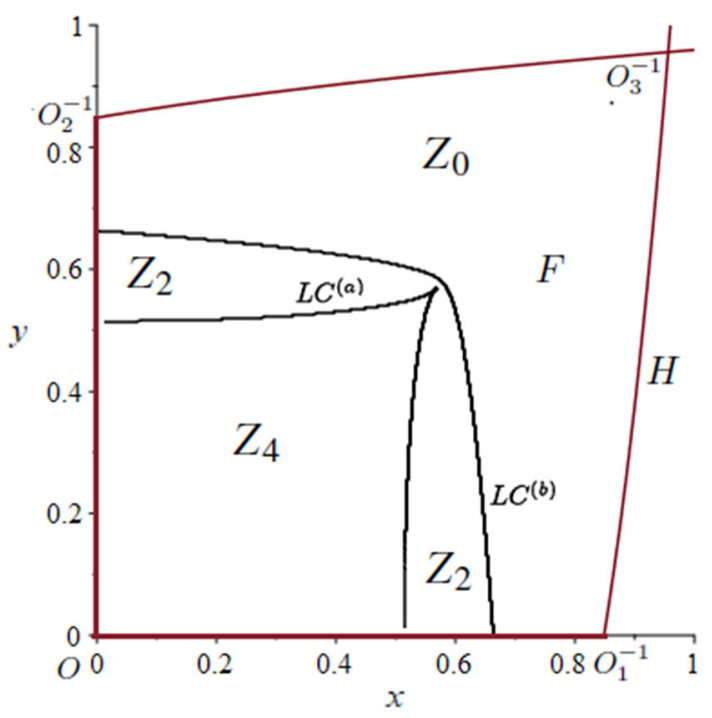

orthant, or (ii) become unfeasible in one iterate, or (iii) reach the fixed point $O=(0,0)$ in one iteration starting from $O_{k}^{-1}$, with $k=1,2$. Here, $O_{k}^{-1}$ is the rank-1 preimage of the origin located along the $x$ (resp. $y$ ) axis when $k=1$ (resp. $k=2$ ) with a non-null coordinate equal to $a_{2}^{\theta /(\theta-1)}$ (resp. $a_{1}^{\theta /(\theta-1)}$ ). In particular, the interior points of the segments $\omega_{k}=\left[O, O_{k}^{-1}\right]$ are points on the boundary of the region $F$ that are reinjected into the interior part of the nonnegative orthant. The rank-1 preimages of such segments form the region $H=O O_{1}^{-1} O_{2}^{-1} O_{3}^{-1}$ shown in Fig. 11 where $O_{3}^{-1}$ is the preimage of $O$ in the interior of the nonnegative orthant. The boundaries of that region thus belong to the boundary of the feasible trajectories. From the study on the number of preimages in the map, we deduce that $\tilde{T}$ is of type $Z_{0}-Z_{2}-Z_{4}$. In the case illustrated in Fig. 11, the preimages $\tilde{T}^{-1}\left(\omega_{k}\right)$ belong to the region $Z_{0}$, that is a region without further preimages. In this case the feasible region $F$ coincides with the region depicted in the figure. For high values of $\theta$, the segments of $H$ and $L C$ become tangent at first and subsequently intersect. In the latter case region $H$ loses its invariance and we witness the emergence of regions (holes), included in $H$, composed of initial conditions that generate unfeasible trajectories.

\subsection{The symmetric case and the problem of synchronization}

We restrict our attention to the symmetric case, in which $a_{1}=a_{2}=a$ and $\lambda_{1}=\lambda_{2}=$ $\lambda$. In this event, the following proposition shows some local dynamic properties of map $\tilde{T}$. 
Proposition 6 Assume $\lambda_{1}=\lambda_{2}=\lambda$ and $a_{1}=a_{2}=a$. Points $\tilde{E}_{0}^{*}=(0,0)$ and $\tilde{E}^{*}=\left(\tilde{x}^{*}, \tilde{x}^{*}\right)$ are fixed points of map $\tilde{T}$, where

$$
\tilde{x}^{*}=\left(\frac{2}{a}\right)^{\theta /(1-\theta)} \times\left(\frac{a}{1+a}\right)^{2 /(1-\theta)}
$$

Moreover, point $\tilde{E}_{0}^{*}$ is always unstable whereas point $\tilde{E}^{*}$ is locally asymptotically stable provided that $a<\tilde{a}_{f}$ and $a<3$, where

$$
\tilde{a}_{f}:=\frac{1}{\lambda}\left(\frac{4}{1-\theta}-\lambda\right)
$$

Proof Imposing stationary conditions $\tilde{T}(x, y)=(x, y)$ and imposing $x=y$, the equation

$$
(1-\lambda) x+\lambda a\left(\left(\frac{2}{a}\right)^{\theta / 2} x^{(\theta+1) / 2}-x\right)-x=0
$$

is obtained, whose solutions are $\tilde{x}_{0}^{*}$ and $\tilde{x}^{*}$. This proves that $\tilde{E}_{0}^{*}$ and $\tilde{E}^{*}$ are stationary states of map $\tilde{T}$. Noting that $\tilde{E}_{0}^{*}, \tilde{E}^{*} \in \Delta$, stability of the fixed points can be evaluated restricting the Jacobian matrix of map $\tilde{T}$ to $\Delta$. It has the structure

$$
\tilde{J}(x, x)=\left(\begin{array}{cc}
\tilde{l}(x) & \tilde{m}(x) \\
\tilde{m}(x) & \tilde{l}(x)
\end{array}\right)
$$

and its eigenvalues are $v_{\|}(x)=\tilde{l}(x)+\tilde{m}(x)$ and $v_{\perp}(x)=\tilde{l}(x)-\tilde{m}(x)$, where $\tilde{l}(x)=$ $1-\lambda+(\lambda a \theta / 4)(2 / a)^{\theta / 2} x^{(\theta-1) / 2}$ and $\tilde{m}(x)=(\lambda a / 2)(2 / a)^{\theta / 2} x^{(\theta-1) / 2}(1+\theta / 2)-$ $\lambda a$. Since $\lim _{x \rightarrow 0} v_{\|}(x)=+\infty$, then $\tilde{E}_{0}^{*}$ is always unstable. The stability conditions $\left|\nu_{\|}\left(\tilde{x}^{*}\right)\right|<1$ related to $\tilde{E}^{*}$ reads as

$$
\left|1-\lambda \frac{1-\theta}{2}-\lambda a \frac{1-\theta}{2}\right|<1
$$

which is satisfied for $a<\tilde{a}_{f}$. At $a=\tilde{a}_{f}, v_{\|}=-1$ holds, namely flip bifurcation occurs. The stability conditions $\left|\nu_{\perp}\left(\tilde{x}^{*}\right)\right|<1$ related to $\tilde{E}^{*}$ read as

$$
\left|1-\lambda+\lambda a-\frac{\lambda}{2}(1+a)\right|<1
$$

which is satisfied for $a<a_{p}:=3$.

Remark 7 Note that $\tilde{x}^{*}$ is a decreasing function of $\theta$ such that $\tilde{x}^{*}=x^{*}$ when $\theta=0$ and $\tilde{x}^{*} \rightarrow 0$ as $\theta \rightarrow 1$. We also remark that condition $a<\tilde{a}_{f}$ ensures that point $\tilde{E}^{*}$ is stable along the direction parallel to $\Delta$, whereas condition $a<3$ ensures that 
Fig. 12 Red points are $(a, \lambda)$ pairs for which $\widetilde{E}^{*}$ is stable given $\theta=0$. As $\theta>0(\theta=0.1$ in the graph), the stability region enlarges in the direction of arrows (color figure online)

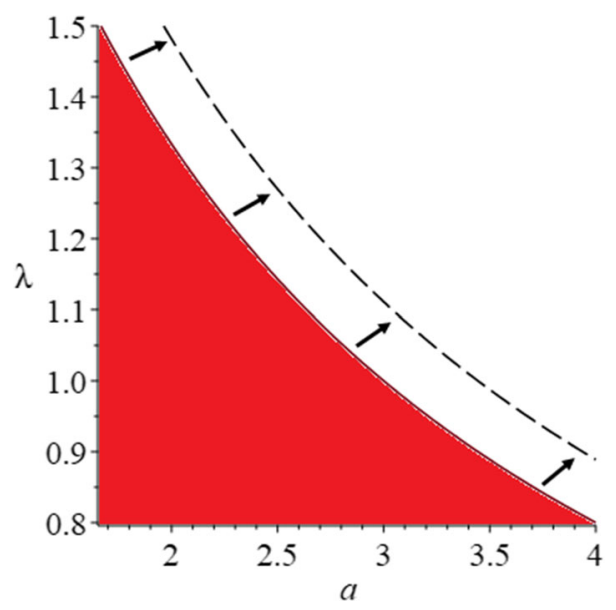

point $\tilde{E}^{*}$ is stable along the transverse direction with respect to $\Delta$. It follows that parameter $\theta$ does not affect the transverse stability of the symmetric fixed point while high values of $\theta$ favor the stability of $\tilde{x}^{*}$ along the direction parallel to $\Delta$. In detail, since $\tilde{a}_{f} \rightarrow+\infty$ as $\theta \rightarrow 1$, the condition $a<\tilde{a}_{f}$ is always satisfied for values of $\theta$ sufficiently close to 1 .

Figure 12 gives a graphical illustration of what is described in Remark 7. Indeed, we may notice that the set of pairs $(a, \lambda)$ that guarantee the stability of the steady state $\tilde{E}^{*}$ becomes larger, as the value of $\theta$ increases from 0 .

Synchronized trajectories are governed by restriction $\tilde{T}_{\Delta}: \Delta \rightarrow \Delta$, where

$$
\tilde{T}_{\Delta}: x^{\prime}=\tilde{f}(x)=(1-\lambda) x+\lambda a\left(\left(\frac{2}{a}\right)^{\theta / 2} x^{(\theta+1) / 2}-x\right)
$$

The following corollary directly follows from Proposition 6.

Corollary 8 Fixed points of map $\tilde{T}_{\Delta}$ are $\tilde{x}_{0}^{*}=0$ and $\tilde{x}^{*}$. Point $\tilde{x}_{0}^{*}=0$ is always unstable, whereas the fixed point $\tilde{x}^{*}$ is locally asymptotically stable provided that $a<\tilde{a}_{f}$. Moreover, point $\tilde{x}^{*}$ undergoes flip bifurcation at $a=\tilde{a}_{f}$.

We restrict the values of parameter $a$ in order to have feasible trajectories of map $\tilde{f}$. The threshold for $a$ is provided in the following proposition together with further characterizations of map $\tilde{f}$. Our aim is to highlight that initial conditions included in the set $\tilde{S}$ generate trajectories included in $\tilde{S}$. Moreover, possible chaotic motions can be observed in $\tilde{S}$ due to the existence of an absorbing set $M \subseteq \tilde{S}$.

Proposition 9 Assume $a \leq \tilde{a}_{\max }$, where

$$
\tilde{a}_{\max }:=\frac{1-\lambda}{\lambda}+\frac{1}{\lambda(1-\theta)}\left(\frac{4}{(\theta+1)^{\theta+1}}\right)^{1 /(1-\theta)}
$$


(a) If $a \in(0,(1-\lambda) / \lambda]$, then $\tilde{f}$ is monotonically increasing in $[0,+\infty)$ with absorbing set $M=\left\{\tilde{x}^{*}\right\}$

(b) if $a \in\left((1-\lambda) / \lambda, \tilde{a}_{\max }\right]$, then $\tilde{f}$ is unimodal in the set $\tilde{S}=\left[0, \tilde{x}_{\max }\right]$, where

$$
\tilde{x}_{\max }:=\left(\frac{\lambda a}{\lambda+\lambda a-1}\right)^{2 /(1-\theta)} \times\left(\frac{2}{a}\right)^{\theta /(1-\theta)}
$$

and an absorbing set $M \subseteq \tilde{S}$ exists.

Proof Map $\tilde{f}$ is concave, which follows from the continuity of $\tilde{f}$ in $[0,+\infty)$ and from the relation $\tilde{f}^{\prime \prime}(x)<0$ that is satisfied for all $x>0$. In addition, $\tilde{f}$ is monotonically increasing in $[0,+\infty)$ when $a \leq(1-\lambda) / \lambda$ since in this case the inequality $\tilde{f}^{\prime}(x)>0$ is satisfied for all $x>0$. The same restriction on $a$ values implies local stability of the fixed point, namely the singleton $M=\left\{\tilde{x}^{*}\right\}$ is absorbing. Differently, if $a>(1-\lambda) / \lambda$, $\tilde{f}$ reach its absolute maximum at the critical point

$$
\tilde{x}_{c r}=\left(\frac{\lambda a(\theta+1)}{2(\lambda+\lambda a-1)}\right)^{2 /(1-\theta)} \times\left(\frac{2}{a}\right)^{\theta /(1-\theta)}
$$

Moreover, the equation $\tilde{f}(x)=0$ is satisfied at 0 as well as at $x_{\max }$. Hence, by concavity, map $\tilde{f}$ is unimodal in $\tilde{S}$. We finally mention that the inequality $\tilde{f}\left(x_{c r}\right) \leq$ $x_{\max }$ is equivalent to $a \leq \tilde{a}_{\max }$, which can be deduced from direct computations. It follows that, if $(1-\lambda) / \lambda<a \leq a_{\max }$, an absorbing set $M$ included in $\tilde{S}$ exists. Also in this, $M=\left\{\tilde{x}^{*}\right\}$ can occur. Alternatively, for values of $a$ beyond the stability threshold $\tilde{a}_{f}$, the absorbing set $M$ can be an interval (as well as union of intervals) bounded by rank $k$-images of the critical point $\tilde{x}_{c r}$, with $k \geq 2$.

\subsubsection{Synchronization achievement and discussion}

The implications that the assumption of endogenous market size has on the possible achievement of synchronization patterns are here investigated. The first indication in this direction originates from observing that when $\theta=0$, map $\tilde{T}$ reduces to $T$. Hence, when $\theta$ is sufficiently close to zero, no abrupt change is expected in global scenarios with respect to those arising when $\theta=0$, since small values of $\theta$ entail the weak dependence of market size on aggregate attraction and, in turn, small step variations of $B$ values. In Fig. 13, complex attractors $A_{s}$ along $\Delta$ (shown in the bifurcation diagram in Panel (a)), observed for $\theta$ varying in a neighborhood of zero, are paired with transverse stability and instability features of $A_{S}$ (as shown by negative and positive values of the Lyapunov exponent $\Lambda_{\perp}$ shown in Panel (b)). Such an alternation of stability property of $A_{s}$ causes global bifurcation scenarios (such as global and local riddling and blowout bifurcations) through the same mechanisms described in the previous sections. A further indication about the effects of endogenous $B$ comes from the role that parameter $\theta$ plays on the stability of $\tilde{E}^{*}$ along the parallel direction to the synchronization manifold. Indeed, in a scenario where a complex attractor is present along $\Delta$ at a given $\theta$, increasing values of this parameter will reduce such complexity, 

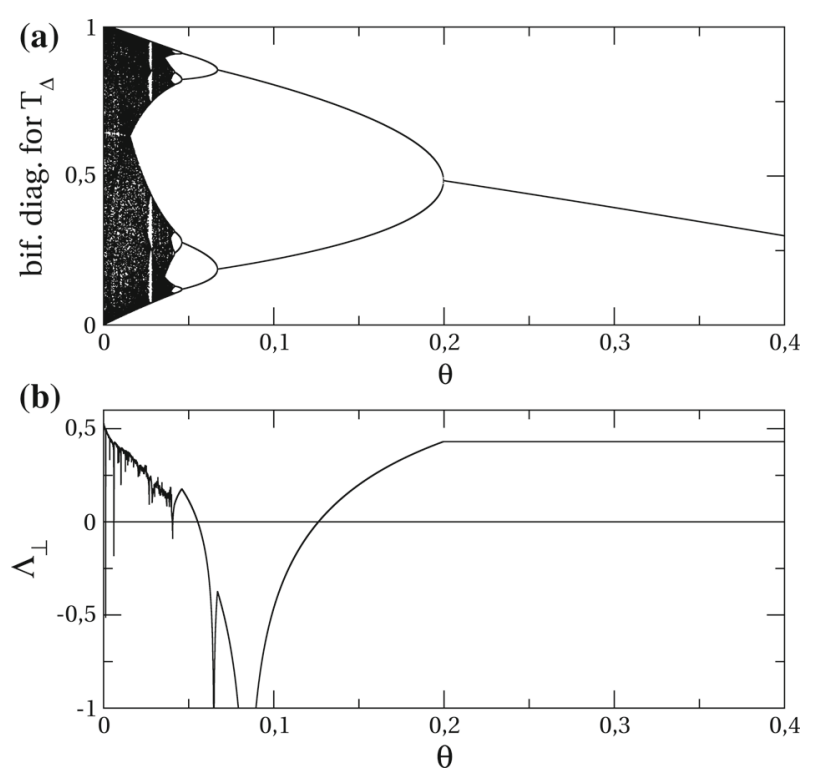

Fig. 13 a Bifurcation diagram of asymptotic dynamics of the restriction $\tilde{T}_{\Delta}$ as $\theta$ increases. b Transverse Lyapunov exponent $\Lambda_{\perp}$ as the dynamics along the synchronization manifold $\Delta$ changes as $\theta$ increases. Common parameters are $\lambda=0.98$ and $a=4.1$; in this case $\tilde{a}_{\max } \sim 4.102$

following the typical period-halving sequence of flip bifurcations, up to reducing the dynamics on $\Delta$ to convergence to the fixed point (see Remark 7). Then, for sufficiently high values of $\theta$, only cycles that are stable along $\Delta$ are present on it. Such cycles are either locally asymptotically stable for the full map $\tilde{T}$ or unstable (saddles) ${ }^{5}$. In the former case, occurrence of synchronized dynamics is a robust scenario that can be observed for sets of initial conditions having positive measures. Moreover, small differences between agents (namely slight differences between parameters $a_{1}$ and $a_{2}$ or $\lambda_{1}$ and $\lambda_{2}$ ) will not determine abrupt changes in global scenarios, and quasi-synchronized states are robust long-run configurations as well. In the latter occurrence, saddle cycles on $\Delta$ can be achieved only for initial conditions on $\Delta$ (a set of zero measure in the plane) and synchronization can be observed only in nongeneric circumstances. In the bifurcation diagram in Fig. 13 Panel (a), only periodic cycles appear on $\Delta$ for about $\theta>0.04$. The Lyapunov exponent $\Lambda_{\perp}$ provided in Panel (b) shows the related transverse attractiveness of the mentioned cycles. We note that, for about $\theta>0.2$, point $\tilde{E}^{*}$ is stable along $\Delta$ and transversely unstable. ${ }^{6}$ Then, the constant and positive value of $\Lambda_{\perp}$ in this range is justified because trajectories starting on $\Delta$ are caught by $\tilde{E}^{*}$.

\footnotetext{
5 besides non-generic cases of local bifurcation scenarios.

${ }^{6}$ In the simulation $a=4.1>3$; we recall that transverse attractiveness is not affected by $\theta$ (see Remark 7). 


\section{Conclusions}

In this article, we considered the map proposed by Bischi and Baiardi (2015a), which is based on the market share attraction model considered in Farris et al. (2005). Furthermore, an extension of the model taking into account possible dependence of the sales potential from customers' attraction (endogenous market size) was formulated. We discussed some properties of the original map and its extension that had not been deepened before. In detail, we addressed the problem of synchronization achievement, studying the transversal attractiveness of the attractor embedded in the invariant synchronization manifold. The analysis was based on well-known results (see, e.g., Alexander et al. 1992; Ashwin et al. 1996; Buescu 2012; Venkataramani et al. 1996; Bischi et al. 1998; Bischi and Gardini 2000; Bischi and Baiardi 2015b and Bischi and Cerboni Baiardi (2017) among others). Through theoretical arguments, paired with numerical simulations, we showed the existence of both multistability even in the presence of homogeneous agents as well as in the presence of non-topological Milnor attractors, which determines the occurrence of non-synchronization patterns both in the short run (on-off intermittency) and in the long run (global riddling). Therefore, the work allowed the authors to notice that there are strategic contexts in which the players' knowledge of the market and the adoption of the best reply do not allow the use of the representative agent's rhetoric to describe the dynamics of the system.

Acknowledgements With this contribution, we take the opportunity to honor our friend Professor Gian Italo Bischi for the attention he devoted to research, dissemination and teaching that involved and guided us over the years with generosity and care. Thank you Gian Italo! Work developed in the framework of the research project on "Models of behavioral economics for sustainable development" financed by DESP-University of Urbino. Mauro Sodini acknowledges the support by VSB-TUO project SP2021/15 and the Science without borders 2.0 project, CZ.02.2.69/0.0/0.0/18_053/0016985 within the Operational Programme Research, Development and Education. We finally thank two anonymous referees for their useful comments.

Funding Open access funding provided by Università della Calabria within the CRUI-CARE Agreement.

Open Access This article is licensed under a Creative Commons Attribution 4.0 International License, which permits use, sharing, adaptation, distribution and reproduction in any medium or format, as long as you give appropriate credit to the original author(s) and the source, provide a link to the Creative Commons licence, and indicate if changes were made. The images or other third party material in this article are included in the article's Creative Commons licence, unless indicated otherwise in a credit line to the material. If material is not included in the article's Creative Commons licence and your intended use is not permitted by statutory regulation or exceeds the permitted use, you will need to obtain permission directly from the copyright holder. To view a copy of this licence, visit http://creativecommons.org/licenses/by/4.0/.

\section{References}

Abraham, R., Mira, C., Gardini, L.: Chaos in Discrete Dynamical Systems. Springer, Berlin (1997) Alexander, J., Yorke, J.A., You, Z., Kan, I.: Riddled basins. Int. J. Bifurc. Chaos 2(04), 795-813 (1992) Aoki, M.: New Approaches to Macroeconomic Modeling: Evolutionary Stochastic Dynamics, Multiple Equilibria, and Externalities as Field Effects. Cambridge University Press, Cambridge (1998)

Ashwin, P., Buescu, J., Stewart, I.: From attractor to chaotic saddle: a tale of transverse instability. Nonlinearity 9(3), 703 (1996) 
Basuroy, S., Nguyen, D.: Multinomial logit market share models: equilibrium characteristics and strategic implications. Manage. Sci. 44(10), 1396-1408 (1998)

Bell, D.E., Keeney, R.L., Little, J.D.: A market share theorem. J. Mark. Res. 12(2), 136-141 (1975)

Bischi, G.I., Baiardi, L.C.: A dynamic marketing model with best reply and inertia. Chaos, Solitons Fractals 79, 145-156 (2015a)

Bischi, G.I., Baiardi, L.C.: Fallacies of composition in nonlinear marketing models. Commun. Nonlinear Sci. Numer. Simul. 20(1), 209-228 (2015b)

Bischi, G.I., Cerboni Baiardi, L.: Bubbling, riddling, blowout and critical curves. J. Differ. Equ. Appl. 23(5), 939-964 (2017)

Bischi, G.I., Gardini, L.: Role of invariant and minimal absorbing areas in chaos synchronization. Phys. Rev. E 58(5), 5710 (1998)

Bischi, G.I., Gardini, L.: Global properties of symmetric competition models with riddling and blowout phenomena. Discret. Dyn. Nat. Soc. 5(3), 149-160 (2000)

Bischi, G.I., Kopel, M.: Multistability and path dependence in a dynamic brand competition model. Chaos, Solitons Fractals 18(3), 561-576 (2003)

Bischi, G.I., and Lamantia, F.: Chaos synchronization and intermittency in a duopoly game with spillover effects. In: Oligopoly Dynamics pp. 195-217. Springer, Berlin (2002a)

Bischi, G.I., Lamantia, F.: Nonlinear duopoly games with positive cost externalities due to spillover effects. Chaos, Solitons Fractals 13(4), 701-721 (2002b)

Bischi, G.I., and Naimzada, A.: Global analysis of a dynamic duopoly game with bounded rationality. In: Advances in Dynamic Games and Applications, pp. 361-385. Springer, Berlin (2000)

Bischi, G.I., Stefanini, L., Gardini, L.: Synchronization, intermittency and critical curves in a duopoly game. Math. Comput. Simul. 44(6), 559-585 (1998)

Bischi, G.I., Gallegati, M., Naimzada, A.: Symmetry-breaking bifurcations and representativefirm in dynamic duopoly games. Ann. Oper. Res. 89, 252-271 (1999)

Bischi, G.I., Gardini, L., Kopel, M.: Analysis of global bifurcations in a market share attraction model. J. Econ. Dyn. Control 24(5-7), 855-879 (2000)

Bischi, G.I., Chiarella, C., Kopel, M., Szidarovszky, F., et al.: Nonlinear Oligopolies. Springer, Berlin (2010)

Bischi, G.I., Lamantia, F., Radi, D.: An evolutionary cournot model with limited market knowledge. J. Econ. Behav. Organ. 116, 219-238 (2015)

Bonanno, G., Zeeman, E.C.: Limited knowledge of demand and oligopoly equilibria. J. Econ. Theory 35(2), 276-283 (1985)

Buescu, J.: Exotic Attractors: From Liapunov Stability to Riddled Basins, Vol. 153. Birkhäuser (2012)

Carpenter, G.S., Cooper, L.G., Hanssens, D.M., Midgley, D.F.: Modeling asymmetric competition. Mark. Sci. 7(4), 393-412 (1988)

Cavalli, F., Naimzada, A., Pireddu, M.: Heterogeneity and the (de) stabilizing role of rationality. Chaos, Solitons Fractals 79, 226-244 (2015)

Cooper, L. G., Nakanishi, M.: Market-Share Analysis: Evaluating Competitive Marketing Effectiveness, Vol. 1. Springer Science \& Business Media, Berlin (1989)

Fanti, L., Gori, L., Sodini, M.: Nonlinear dynamics in a cournot duopoly with isoelastic demand. Math. Comput. Simul. 108, 129-143 (2015)

Farris, P., Pfeifer, P.E., Reibstein, D., van Nierop, E.: When five is a crowd in the market share attraction model. Marketing ZFP, 27(JRM 1):41 (2005)

Federgruen, A., Yang, N.: Competition under generalized attraction models: applications to quality competition under yield uncertainty. Manage. Sci. 55(12), 2028-2043 (2009)

Fujisaka, H., Yamada, T.: Stability theory of synchronized motion in coupled-oscillator systems. Progress Theoret. Phys. 69(1), 32-47 (1983)

Gumowski, I., Mira, C.: Dynamique chaotique: transformations ponctuelles, transition, ordre-désordre (1980)

Hommes, C.H., LeBaron, B.D.: Handbook of Computational Economics. North Holland, Amsterdam (2018)

Kapitaniak, T.: Partially nearly riddled basins in systems with chaotic saddle. Chaos, Solitons Fractals 12(13), 2363-2367 (2001)

Karnani, A.: Strategic implications of market share attraction models. Manage. Sci. 31(5), 536-547 (1985)

Kirman, A.P.: Whom or what does the representative individual represent? J. Econ. Perspect. 6(2), 117-136 (1992)

Kopel, M.: Simple and complex adjustment dynamics in cournot duopoly models. Chaos, Solitons Fractals 7(12), 2031-2048 (1996) 
Lai, Y.-C., Grebogi, C., Yorke, J.A., Venkataramani, S.: Riddling bifurcation in chaotic dynamical systems. Phys. Rev. Lett. 77(1), 55 (1996)

Maistrenko, Y., Kapitaniak, T., Szuminski, P.: Locally and globally riddled basins in two coupled piecewiselinear maps. Phys. Rev. E 56(6), 6393 (1997)

Maistrenko, Y.L., Maistrenko, V., Popovich, A., Mosekilde, E.: Role of the absorbing area in chaotic synchronization. Phys. Rev. Lett. 80(8), 1638 (1998a)

Maistrenko, Y.L., Maistrenko, V., Popovich, A., Mosekilde, E.: Transverse instability and riddled basins in a system of two coupled logistic maps. Phys. Rev. E 57(3), 2713 (1998b)

Massaro, D.: Heterogeneous expectations in monetary dsge models. J. Econ. Dyn. Control 37(3), 680-692 (2013)

Milnor, J.: On the concept of attractor. In: The Theory of Chaotic Attractors, pp. 243-264. Springer, Berlin (1985)

Mira, C., Gardini, L., Barugola, A., Cathala, J.-C.: Chaotic Dynamics in Two-Dimensional Noninvertible Maps, vol. 20. World Scientific, Singapore (1996)

Neumann, E., Sushko, I., Maistrenko, Y., Feudel, U.: Synchronization and desynchronization under the influence of quasiperiodic forcing. Phys. Rev. E 67(2), 026202 (2003)

Pecora, L.M., Carroll, T.L.: Synchronization in chaotic systems. Phys. Rev. Lett. 64(8), 821 (1990)

Popovych, O., Maistrenko, Y., Mosekilde, E., Pikovsky, A., Kurths, J.: Transcritical loss of synchronization in coupled chaotic systems. Phys. Lett. A 275(5-6), 401-406 (2000)

Popovych, O., Maistrenko, Y., Mosekilde, E.: Loss of coherence in a system of globally coupled maps. Phys. Rev. E 64(2), 026205 (2001)

Puu, T.: Chaos in duopoly pricing. Chaos, Solitons Fractals 1(6), 573-581 (1991)

Venkataramani, S.C., Hunt, B.R., Ott, E.: Bubbling transition. Phys. Rev. E 54(2), 1346 (1996)

Zimin, A.V., Hunt, B.R., Ott, E.: Bifurcation scenarios for bubbling transition. Phys. Rev. E 67(1), 016204 (2003)

Publisher's Note Springer Nature remains neutral with regard to jurisdictional claims in published maps and institutional affiliations. 